Mots. Les langages du politique

$111 \mid 2016$

Normes et usages de la langue en politique

\title{
Les usages du qualificatif droitier au Nouveau Parti anticapitaliste. Orthodoxie et pluralisme intrapartisan à l'extrême gauche
}

The uses of the adjective right wing in New Anti-Capitalist Party. Orthodoxy and intra-partisan pluralism in the ranks of the radical left Los usos del qualificativo droitier en el Nouveau Parti anticapitaliste. Ortodoxia y pluralismo intra-partisano en la extrema izquierda

Jérémy Sinigaglia

\section{OpenEdition \\ Journals}

Édition électronique

URL : https://journals.openedition.org/mots/22389

DOI : $10.4000 /$ mots. 22389

ISBN : 978-2-84788-836-2

ISSN : 1960-6001

Éditeur

ENS Éditions

\section{Édition imprimée}

Date de publication : 10 septembre 2016

Pagination : 103-120

ISBN : 978-2-84788-835-5

ISSN : 0243-6450

Référence électronique

Jérémy Sinigaglia, « Les usages du qualificatif droitier au Nouveau Parti anticapitaliste. Orthodoxie et pluralisme intrapartisan à l'extrême gauche », Mots. Les langages du politique [En ligne], 111 | 2016, mis en ligne le 10 septembre 2018, consulté le 22 avril 2022. URL : http://journals.openedition.org/mots/ 22389 ; DOI : https://doi.org/10.4000/mots.22389 


\title{
Les usages du qualificatif droitier au Nouveau Parti anticapitaliste. Orthodoxie et pluralisme intrapartisan à l'extrême gauche
}

\begin{abstract}
"La lutte intérieure donne au parti la force et la vitalité : la preuve, la plus grande de la faiblesse du parti, c'est son amorphisme et l'absence de frontières nettement délimitées; le parti se renforce en s'épurant. » Extrait d'une lettre de Lassalle à Marx, 24 juin 1852, cité par Lénine en ouverture de la préface de Que faire?
\end{abstract}

Le clivage gauche/droite, qui trouve son origine dans la topographie parlementaire avant de se diffuser plus largement à partir du début du $20^{\mathrm{e}}$ siècle (Gauchet, 1997; Crapez, 1998), reste, en dépit des interrogations récurrentes dont il fait l'objet (Aebischer, 2003), un principe fort de division du monde politique, un instrument de classement des idéologies, des partis et des agents au sein du champ politique (Le Bohec, Le Digol, 2012). Mais ce clivage «pluriel» (Richard, 2006) n'est pas seulement mobilisé dans les relations interpartisanes : les notions de gauche et de droite, toujours relatives, fonctionnent également comme un principe de division à l'intérieur des partis eux-mêmes, au sein desquels se rejouent les luttes de position et les stratégies de distinction. L'histoire de l'extrême gauche en général, et des trotskismes en particulier (Bensaïd, 2002), en fournit une excellente illustration. Cette topographie politique interne est déjà présente dans les écrits de Lénine et de Trotsky, distinguant les «ultra-gauchistes» (anarchistes, anarcho-syndicalistes, blanquistes), les "gauchistes» (spartakistes, bordiguistes) mais aussi les «centristes» et les «droitiers» (opportunistes, réformistes), tous qualifiés de «déviationnistes» par rapport à la ligne bolchevique ${ }^{1}$. Parmi ces catégories, c'est celle de droitier

1. Voir notamment Lénine, La Maladie infantile du communisme. Le gauchisme, 1920 : 〈http:// www.marxists.org/francais/lenin/works/1920/04/gauchisme.htm> (consulté le 16 mars 2016, de même que tous les sites mentionnés dans les notes) ; Trotsky, «Les erreurs des éléments droitiers de la Ligue dans la question syndicale », E Fuvres, 1931 : 〈http://www.marxists.org/francais/trotsky/oeuvres/1931/01/lt19310104.htm〉; Trotsky, «Bolchévisme contre stalinisme», Euvres, 1937, 〈http://www.marxists.org/francais/trotsky/livres/bcs/bcs05.htm〉

IEP de Strasbourg, SAGE

jeremy.sinigaglia@unistra.fr 
qui a retenu notre attention, à la fois pour son incongruité apparente (qu'estce qu'être droitier à l'extrême gauche?) et pour sa réapparition dans les débats contemporains, notamment au sein du Nouveau parti anticapitaliste (NPA) issu de la dissolution, en 2009, de la Ligue communiste révolutionnaire (Johsua, 2015). La recomposition en cours de l'extrême gauche donne l'opportunité de réinterroger la consistance et le rôle de ces étiquetages, particulièrement celui de droitier, qui tient une place particulière dans l'histoire du mouvement ouvrier, puisqu'on le retrouve aussi bien dans les procès staliniens, dits du «Bloc des droitiers et des trotskistes antisoviétiques» (Werth, 2006), que dans l'histoire des scissions trotskistes en France (Lindenberg, 2004). Si les textes adoptés au congrès de fondation du NPA donnent au parti l'objectif de «faire vivre l'héritage de celles et ceux qui ont affronté le système depuis deux siècles, celui de la lutte des classes, des traditions socialistes, communistes, libertaires, révolutionnaires $»^{2}$ ou, pour reprendre une formule employée par son porte-parole de l'époque, Olivier Besancenot, de réunir «le meilleur des traditions du mouvement ouvrier »3, et affirment la possibilité d'une hétérogénéité et d'un débat démocratique internes garantis par «le droit de tendance et le droit de fraction »4, l'arrivée de néomilitants et surtout de militants socialisés politiquement dans d'autres courants génère de fortes tensions.

À quoi renvoie alors l'usage du qualificatif droitier? Le comprendre suppose d'abord d'identifier les éléments sur lesquels reposent les classements, tant en termes programmatiques que stratégiques. Mais cela suppose surtout d'analyser la manière dont ces opérations de classement sont mobilisées en pratique, dans les interactions militantes. L'hypothèse est que l'usage de ce qualificatif est un moyen de disqualifier des orientations politiques considérées comme incompatibles avec un programme et une stratégie anticapitalistes et révolutionnaires, et de stigmatiser les militants qui les portent. Mobilisé par les tenants d'une forme d'orthodoxie militante, se revendiquant plus ou moins fortement du trotskisme, le terme témoigne de la (re)construction d'une identité politique valorisant les caractéristiques traditionnelles (et parfois fantasmées) de l'extrême gauche. Mobilisé, de manière ironique, par ceux qui se trouvent ainsi désignés, il renvoie à une stratégie de retournement du stigmate (Goffman, 1977), comme celle qu'ont essayé de mener les membres du courant «anarcho-droitier» du NPA, à l'origine d'un blog satirique destiné à porter le débat au sein de l'organisation5.

2. «Principes fondateurs du Nouveau parti anticapitaliste adoptés par le congrès », février 2009.

3. Dépêche AFP du 14 janvier 2009.

4. "Statuts provisoires du Nouveau parti anticapitaliste adoptés par le congrès », février 2009.

5. Cet article s'appuie sur un travail de recherche qui interroge les dynamiques de recomposition de la gauche radicale en France. L'enquête repose sur trois sources principales : l'observation des activités d'un comité local et du Conseil politique national du Nouveau parti anticapitaliste entre 2007 et 2011 ; un corpus d'archives comprenant l'ensemble des textes officiels (c'est-à-dire les textes soumis au vote au congrès de fondation de février 2009 , les résolutions votées pour les 


\section{La qualification de droitier dans les luttes de positions internes}

Si le terme est absent des principaux textes officiels, dont l'objectif est de fournir un socle commun aux militants, il apparaît dans un certain nombre d'écrits internes et surtout dans des interactions militantes. Dans tous les cas, la qualification de droitier s'apparente à un processus de stigmatisation (Goffman, 1977), désignant comme déviant tout militant qui s'écarte de la doxa, c'est-àdire d'un «ensemble de croyances fondamentales qui n'ont même pas besoin de s'affirmer sous la forme d'un dogme explicite et conscient de lui-même» (Bourdieu, 2003, p. 30). Le qualificatif renvoie ainsi aux luttes de position et aux concurrences qui existent à l'intérieur de l'entreprise politique pour «la définition des fins et des moyens légitimes» (Bourdieu, 1984). Qualifier un argument ou un militant de «droitier», c'est lui donner une position (voire une direction) et c'est aussi se positionner par rapport à lui et, de manière plus globale, au sein du parti6.

\section{Une accusation de «déviationnisme»}

Le terme droitier est mobilisé principalement par des militants - plutôt des hommes, compte tenu de la division sexuelle du travail militant 7 - se revendiquant du trotskisme (ou plutôt d'une de ses branches), sans distinction d'âge ou de génération politique. Cela peut concerner des militants plutôt jeunes, de moins de quarante ans en moyenne, bien dotés en capital culturel (des étudiants du comité de l'École normale supérieure par exemple) et/ou socialisés politiquement dans des organisations de jeunesse de l'extrême gauche (les Jeunesses communistes révolutionnaires en particulier); cela concerne également des ouvriers ou des employés, en moyenne plus âgés et plus fréquemment d'origine populaire, militants de longue date dans des organisations d'extrême gauche (la

élections européennes de 2009 et les élections régionales de 2010 et les divers textes soumis à la discussion et au vote lors des deux premiers congrès, en février 2011 et janvier 2013), des bulletins de discussion de congrès (c'est-à-dire des prises de positions individuelles ou collectives, au niveau d'un comité local par exemple, généralement sur une dimension d'un texte soumis à la discussion) et des billets publiés sur le blog du «courant anarcho-droitier du NPA» entre 2007 et 2013 ; une dizaine d'entretiens avec des militants et ex-militants du NPA, réalisés en 2013.

6. Sur ce double usage des catégories de gauche et de droite, voir Gaxie, 2012.

7. Les rares travaux qui interrogent le travail militant sous l'angle du genre montrent que l'on retrouve, y compris dans les mouvements dits progressistes, une division traditionnelle des tâches : les hommes prennent plus souvent en charge les activités publiques et formalisées (production des mots d'ordre, représentation du groupe) que les femmes, davantage cantonnées aux activités de soutien à l'action militante (Fillieule, Mathieu, Roux, 2007; Dunezat, 2008). Si les militants du NPA sont attentifs à ce que les femmes soient représentées au moins à parité dans la plupart des tâches de direction (composition du Conseil politique national et du bureau, désignation des porte-parole, etc.), les activités quotidiennes ou plus informelles manifestent néanmoins encore une invisibilisation des femmes. 
Ligue communiste révolutionnaire ou Lutte ouvrière par exemple) et/ou dans le mouvement syndical (généralement à la CGT, parfois à SUD).

Le terme apparaît pour la première fois à l'écrit dans une motion proposée par un militant de la tendance CLAIRE8 à l'occasion d'un débat interne sur les élections régionales de 2010. Appelant à la constitution d'une «grande tendance révolutionnaire » au sein du NPA, le texte accuse la direction du parti d'être responsable d'une «dérive droitière et électoraliste " 9 . Dans les mois qui suivent, les prises de position des membres de ce courant politique visent toujours la direction du parti mais également, de plus en plus, les militants dits «unitaires», souhaitant développer les relations politiques avec le Front de gauche nouvellement créé :

C'est ce que font d'ailleurs les courants droitiers assumés [...] aussi bien que les différentes composantes de la majorité [...] Face à la droite assumée du NPA et à l'orientation pro-réformiste de la direction, il faut rassembler les partisans d'un NPA révolutionnaire sur la base d'un programme alternatif. II nous semble évident que, pour contrer l'orientation des droitiers assumés du parti et de la direction, on ne saurait se contenter d'un retour aux textes du congrès fondateur, comme le proposent certains de nos camarades de l'ex-position $\mathrm{B}^{10}$. Ce serait en effet revenir à une étape complètement dépassée par la réalité et ne pas se hisser à la hauteur des enjeux posés par la direction, dont la politique a conduit à la crise du NPA. ${ }^{11}$

Il était pour nous indispensable de répondre à la politique de la direction et aux éléments droitiers du NPA qui avaient mis le parti dans une impasse. Il était nécessaire pour nous de mener un combat sur le plan idéologique et politique pour que le NPA soit un parti révolutionnaire, un parti qui assimile la tradition des grands penseurs marxistes révolutionnaires, de Marx, Engels, Rosa Luxembourg, Lénine et Trotsky,

8. Courant interne du NPA pour «le Communisme, la Lutte Auto-organisée, Internationaliste et RévolutionnairE ». Il s'agit d'un petit groupe de militants issus du collectif trotskiste Communiste révolutionnaire internationaliste (CRI), créé en 2002 en opposition aux principaux partis trotskistes français (Lutte ouvrière, Ligue communiste révolutionnaire, Parti des travailleurs), qu'ils jugent insuffisamment radicaux.

9. Nicolas (tendance CLAIRE), «Pour un bloc des gauches dans le parti, vers une grande tendance révolutionnaire », motion pour la conférence nationale Jeunes, Bulletin de discussion $n^{\circ} 2$, octobre 2009.

10. Au cours de la consultation nationale pour définir le positionnement du NPA aux élections régionales de 2010, trois positions se sont exprimées et ont été soumises au vote des adhérents. La position A, qui réunit alors les principaux membres de la direction du parti (dont certains finiront quelques mois plus tard par constituer une autre tendance, qui rejoindra le Front de gauche), se revendique des textes fondateurs du parti et propose de conditionner la constitution de listes unitaires avec le Front de gauche à l'accord de ses militants, localement, sur une liste conséquente de revendications; les tenants de la position $B$, parmi lesquels on trouve les animateurs de la tendance CLAIRE mais aussi d'anciens militants de la LCR, considèrent qu'il est impossible de trouver un accord satisfaisant avec les autres composantes de la gauche et proposent de présenter des listes NPA, ouvertes à des «militants du mouvement social», partout où cela est possible; les tenants de la position $C$ souhaitent parvenir à constituer des accords pour des listes dites « unitaires» dont le contenu et les modalités seraient à négocier en fonction des rapports de force locaux.

11. CollectifTR, «Contribution de 62 militants pour une Tendance révolutionnaire du NPA», Réunion nationale de la position B du 23 mai 2010, 17 mai 2010. 
et qui soit un réel outil dans les mains des travailleurs conscients pour renverser le capitalisme et détruire son État, afin de constituer leur propre gouvernement. ${ }^{12}$

Comme l'illustrent ces quelques exemples, l'appellation de droitier est mobilisée principalement dans des débats dits stratégiques, dans lesquels l'objet de la controverse est moins la fin que les moyens de la lutte. De manière récurrente dans les débats de l'extrême gauche, le point de cristallisation des divergences est le rapport aux institutions politiques : faut-il les renverser (faire « du passé table rase ", comme dit la chanson) ou les transformer? Faut-il les contester de l'extérieur ou de l'intérieur? Faut-il participer ou non aux élections? Peuton s'allier à d'autres forces politiques? Dans quelles conditions? Les «éléments droitiers» du parti, considérés comme tels par les « révolutionnaires» (autodésignés), sont ceux qui adoptent ou tendent vers une posture qualifiée de réformiste (ou électoraliste). Lors du débat sur les conditions de la participation aux élections régionales par exemple, la décision de la direction du NPA d'entrer en négociation avec le Parti de gauche pour réfléchir à la possibilité d'une démarche unitaire apparaît déjà comme un compromis inacceptable aux yeux des militants revendiquant une stratégie révolutionnaire. À plus forte raison, les rapprochements entre certains militants du NPA et le Front de gauche sont perçus comme une véritable «dérive ». On retrouve ici une des caractéristiques lexicales récurrentes de l'analyse léniniste, telle qu'elle est exposée notamment dans Que faire? : tout écart par rapport à l'orthodoxie, c'est-à-dire à la « ligne » définie par les cadres du parti, est considéré comme une « déviation » contre laquelle il faut lutter pour faire retrouver aux militants égarés le «droit chemin » (Barbet, 2002). Dans les débats contemporains, on a pu relever un ensemble d'expressions illustrant cette notion d'orientation politique : « le parti n'a [ou les militants n'ont] plus de boussole», «on a perdu le cap », «il faut retrouver le sens de la marche», etc.

\section{Droitier dans les échanges oraux : un terme «guillotine»}

Le qualificatif droitier est également utilisé à l'oral, dans les diverses interactions militantes suscitées par l'activité partisane, dans ou en marge des réunions locales et nationales. Son usage n'est cependant jamais anodin. Comme le fait remarquer un militant interrogé, on lui préfère généralement des termes moins polémiques :

En général, ceux dont on aurait pu attendre ce genre de compliment utilisaient soit des termes plus diplomatiques (s'il s'agissait de convaincre) soit des termes plus spécifiques (s'il s'agissait de cliver : «liquidateurs», « ligne confuse », etc.). ${ }^{13}$

12. Vincent Duse et Manuel Goerget, «Une plateforme révolutionnaire est née dans le NPA », en ligne sur le site de la Fraction trotskiste de la quatrième internationale (FTQI), mai 2010.

13. Pierre, 36 ans, mail du 10 juillet 2013. Précédemment membre d'un ancien courant unitaire du NPA ayant rejoint le Front de gauche. 
Et pour cause, dans les rangs de l'extrême gauche, et en particulier chez les trotskistes, droitier est assimilé à une insulte définissant une «identité par la négative» (Lanuque, 2005, p.173) : dévier ou dériver vers la droite ${ }^{14}$, c'est tendre vers le changement de camp et, à terme, devenir un adversaire de son propre parti et, surtout, un ennemi de sa classe. Cet étiquetage est donc vécu comme une agression par les militants concernés. C'est ce que laisse apparaître le texte d'une motion présentée au congrès de 2010 par des adhérents du comité NPA de l'Yonne :

Le NPA de l'Yonne peut témoigner de l'impossibilité qu'il y a à travailler et construire notre parti avec les militants se réclamant de la tendance CLAIRE et venus du CRI ou en ayant été proches par le passé. N'ayant pas voté les principes fondateurs du NPA, ces militants se sont rapidement positionnés en opposition systématique de toutes les décisions prises, gênant, voire empêchant des initiatives produisant des documents qui ne correspondaient en rien aux orientations des militants icaunais. Cette attitude se trouvait particulièrement exacerbée à l'occasion des assemblées générales qui ne pouvaient se tenir sereinement et se terminant parfois en insulte : l'un de nos camarades a été traité de pétainiste... Ensuite les débats se sont déplacés sur les listes internet où les invectives et les insultes pleuvaient. [...] Quiconque les critiquait était impitoyablement traité de droitier ou de stalinien. [...] Il y a eu sur ces deux pôles, une sorte de mainmise où ils se sont répartis, et ont paralysé ces comités en utilisant des méthodes identiques : attaque des personnes, comptes rendus qui n'étaient pas le reflet des réunions, diffusion de leur point de vue, pas de propositions mais des contestations agressives, postures NPA jugées et qualifiées de droitières.

L'usage oral du qualificatif droitier s'inscrit donc plus généralement dans le contexte d'interactions conflictuelles. Ce n'est pas seulement un terme qui correspond à une grille d'analyse de la vie politique ; il renvoie ici plus directement à une stratégie de stigmatisation, visant à jeter le discrédit sur des arguments ou des positions politiques, mais aussi sur ceux qui les portent. À ce titre, le passage de l'adjectif au substantif est intéressant : ce ne sont pas seulement les arguments qui sont droitiers, ce sont les militants eux-mêmes. Il est d'ailleurs intéressant de noter que le débat réforme/révolution n'est pas adossé, ou seulement de manière très vague, à des références théoriques (comme dans l'extrait cité plus haut) mais à des classements sociaux : selon les « révolutionnaires», le comportement droitier serait celui des «classes moyennes timorées», «d'intellectuels petits bourgeois » ${ }^{15}$. L'explication des positions politiques par l'origine et la position sociales n'est toutefois pas systématique, comme en témoigne ce militant, commentant l'attitude d'une partie de ses camarades lors du départ d'un adhérent qualifié de droitier:

14. C'est le sens «politique» de droitier retenu par dictionnaire de l'Académie : «2. POLIT. Relatifà la fraction la plus modérée d'une formation de gauche. La dérive droitière d'un parti. Subst. Les droitiers d'un parti», 〈http://circe.atilf.fr/definition/academieg/droitier〉.

15. Membre de la tendance CLAIRE, réunion du Conseil politique national. Notes d'observation du 19 septembre 2009, Université de Saint-Denis. 
Le fait de se réjouir du départ d'un camarade droitier, c'est parce qu'en l'occurrence il est pas ouvrier chez Peugeot! Parce qu'il aurait été droitier et ouvrier chez Peugeot, d'abord il aurait peut-être pas été traité de droitier... et la question est posée... à quelle classe sociale il appartient? Moi j'ai déjà tenu des positions ou des propos qu'on pourrait qualifier de droitiers, mais comme j'étais un ouvrier, un travailleur, il y avait un peu de condescendance à mon égard, je le sentais bien... en disant bon, il raconte des conneries mais on va être gentil avec lui... au niveau national surtout, oui, oui... bien sûr... sauf ceux qui me connaissaient pas, ils te rentrent dedans, mais ceux qui savent...16

Le terme n'est donc pas seulement un élément de caractérisation d'une position dans le débat, il permet de mettre l'autre à distance. L'insulte est en effet, comme le rappelle Pierre Bourdieu, un acte d'institution autant que de «destitution » : elle attribue des propriétés particulières à un individu et l'invite à « se comporter en conformité avec l'essence sociale qui lui est ainsi assignée» (Bourdieu, 2001, p.156). L'usage du qualificatif droitier s'inscrit, selon l'expression utilisée par un militant, dans un « débat fractionnel » : l'enjeu n'est pas de mettre l'accent sur une divergence d'analyse en pensant qu'il est possible de convaincre l'autre de son erreur, mais bien de tracer une frontière entre deux conceptions politiques jugées incompatibles et irréconciliables. Dans ce contexte, comme le notent ces militants, droitier est un « argument guillotine», «une façon de couper la discussion» :

Droitier, en fait, c'est un terme très guillotine. Quand t'es en débat avec quelqu'un, c'est l'argument guillotine, ça coupe court à toute discussion : c'est droitier donc c'est pas valable. [...] Quand le terme est sorti, en débat, c'est guillotine parce que après tu te justifies sur pourquoi c'est pas droitier, et tu parles plus du débat en luimême... C'est une diversion quoi. ${ }^{17}$

En général, si tu traites l'autre de droitier, c'est que le débat a monté d'un ton, au moins sur la forme... Mais c'est aussi une façon de couper la discussion : on ne peut pas aller plus loin parce que toi t'es un droitier! Et donc après ça simplifie les débats, enfin en tout cas ça les règle de cette façon-là... Tu vois, avec l'exemple du camarade, sa position ou ses interrogations sur l'avortement, et ben y'a pas de débat, «sa position elle est droitière », point, c'est terminé. Pas besoin d'argumenter. ${ }^{18}$

L'enjeu de ce type d'interactions est double. D'un côté, il s'agit de produire des identités politiques. Chacun met en avant des éléments qu'il estime le caractériser d'un point de vue militant, et est perçu et désigné à son tour par les personnes avec lesquelles il entre en relation. S'engagent alors des négociations, ou plutôt des rapports de force, qui détermineront qui, de l'identité pour soi ou pour autrui (Dubar, 2000), l'emporte. D’un autre côté, il s'agit de

16. Jean-Claude, ouvrier cheminot à la retraite, 65 ans, entretien du 17 juillet 2013.

17. Guillaume, 35 ans, enseignant contractuel, entretien du 18 juillet 2013.

18. Jean-Claude, ouvrier cheminot à la retraite, 65 ans, entretien du 17 juillet 2013. 
produire le sens de l'action collective. Les tenants des différentes positions sont en concurrence pour déterminer la « ligne » de l'organisation, c'est-à-dire la conduite politique légitime à laquelle chacun sera ensuite appelé à se conformer. Dans ce jeu d'interactions, la qualification de droitier joue un rôle important puisqu'elle conduit à disqualifier un argument et ceux qui le soutiennent. La métaphore de la "guillotine» utilisée par les militants est révélatrice du degré de disqualification que produit cette étiquette, même si elle est utilisée prioritairement par les tenants des positions les plus radicales, minoritaires au sein de l'organisation (comme CLAIRE) : elle invite à rompre la discussion, mais aussi à prendre ses distances avec les interlocuteurs indignes. Dans les moments de tensions internes au parti, les membres de la tendance majoritaire ou d'autres courants distincts de la tendance CLAIRE ont ainsi rejoint leurs arguments (par exemple, la critique de «l'angélisme» des militants dits " unitaires », qui feraient preuve de «naïveté politique » en cherchant à nouer des alliances à «n'importe quel prix»)19, ce que l'on peut analyser en partie 20 comme le refus d'être associé d'une manière ou d'une autre à une position droitière. Contrairement à l'étiquette a priori opposée de "gauchiste », qui est sinon revendiquée, au moins assumée (parfois avec humour), celle de droitier est à même de faire «perdre la face». En ce sens, elle peut constituer une sorte d' « incitation négative» (Olson, 1978) favorisant, plus ou moins consciemment, l'«orthodoxie militante» (Gaxie, 1977) : il faut éviter, individuellement et collectivement, les prises de position qui présentent le risque de se voir attribuer l'étiquette infamante de droitier. Ce stigmate, au sens fort de la «différence honteuse» (Goffman, 1977), se révèle particulièrement puissant et difficile à détourner en attribut positif, comme le montre l'expérience qui suit.

\section{Une tentative échouée de retournement du stigmate : le courant anarcho-droitier du NPA}

Dans un temps très court, le NPA a donné l'illustration d'au moins deux phénomènes politiques classiques et en partie liés. Le premier concerne les « inconstances politiques» (Gaïti, 2001) : de nombreux militants aux trajectoires variées ont changé d'opinion, que ce soit sur des questions programmatiques ou stratégiques, des sujets centraux ou périphériques. Cela s'est traduit par une multiplication des courants internes, organisés autour d'une conception particulière de l'action politique, illustrant ce deuxième phénomène qu'est la propension historique aux scissions de la gauche anticapitaliste (De Waele, Seiler, 2011).

19. Réunion d'un comité local du NPA. Notes d'observation du 10 décembre 2009.

20. En partie seulement, car l'accord sur l'analyse ne tient pas qu'aux interactions, il s'explique aussi par les socialisations politiques des uns et des autres, et notamment par l'ancienneté et le degré d'implication dans des formations de l'extrême gauche trotskiste. 
En quatre ans, le NPA est passé d'un parti organisé relativement uni autour des mêmes principes fondateurs (avec un peu plus de 9000 adhérents) à une organisation divisée en cinq positions au congrès de 2013 (pour un peu moins de 2000 adhérents), auxquelles on peut ajouter les trois tendances qui l'ont successivement quitté pour rejoindre le Front de gauche. À ces différents courants, il aurait pu s'en ajouter un autre. À mi-chemin entre démarche critique et purement satirique, ses animateurs ont voulu se réapproprier le qualificatif droitier pour en atténuer la portée et tenter de dépasser les clivages traditionnels de l'extrême gauche.

\section{La naissance du « blog du courant anarcho-droitier du NPA »}

Le projet de créer un courant «anarcho-droitier» au sein du NPA a germé en 2011 dans la tête de Guillaume, un enseignant âgé de 35 ans, membre du parti depuis sa création après avoir rejoint, en 2008 et sans rien savoir de la dissolution qui se préparait, la Ligue communiste révolutionnaire. Ancien membre du Parti communiste français (de 18 à 22 ans), puis militant de la Fédération syndicale étudiante (il y occupe diverses responsabilités locales puis nationales), il est au départ très enthousiaste devant la promesse de «nouveauté » que porte le NPA. Mais au fil des mois, il regrette ce qu'il analyse comme une forme de repli sur soi contraire à l'esprit d'ouverture revendiqué à la fondation du parti. Dans un premier temps, il envisage de constituer une tendance au sein du NPA, mais il comprend rapidement qu'il manque des ressources politiques et relationnelles pour le faire. Avec un de ses amis, militant dans le même comité local que lui, il décide alors d'ouvrir un blog «critique, ironique et humoristique » intitulé «Fight Club²1 : le blog du courant anarcho-droitier du NPA».

Le choix du nom, argumenté dans le billet d'ouverture du blog²2, repose précisément sur l'expérience vécue de la qualification de droitier. Pour Guillaume, c'est le ralliement à la plateforme « unitaire ${ }^{23}$, qui défendait la poursuite des

21. En référence au film éponyme de David Fincher (1999) sur l'organisation de combats clandestins particulièrement violents.

22. "Charte du courant anarcho-droitier», billet du 31 juillet 2011.

23. Un congrès du NPA est organisé autour d'une série de «plateformes », c'est-à-dire de textes qui portent des points de vue concurrents sur l'analyse de la situation politique et sociale et sur la stratégie à suivre; ces plateformes peuvent donner lieu à la constitution de tendances à l'intérieur du parti. Au congrès de janvier 2011, quatre plateformes, issues de recompositions des positions exprimées lors des élections régionales de 2010 (voir note 10), sont soumises à la discussion : la première $\left(\mathrm{P}_{1}\right)$, dite «majo», est présentée par des membres de la direction (une partie de l'ancienne position $A$ ) ; la seconde $\left(\mathrm{P}_{2}\right)$ regroupe les promoteurs d'une ligne plus radicale (une partie de l'ancienne position $B$ ) ; la troisième $\left(P_{3}\right)$, dite « unitaire», souhaite un rapprochement avec le Front de gauche (une partie de l'ancienne position $C$ - ceux qui n'ont pas encore quitté le NPA - et d'anciens dirigeants de la position $A)$; la dernière ( $\left.P_{4}\right)$, est issue d'une scission de l'ancienne position $B$ et défend une ligne plus explicitement « révolutionnaire » (autour de la tendance CLAIRE notamment). 
discussions avec le Front de gauche dans le cadre des élections régionales, qui lui a valu d'être désigné ainsi, «d'abord sur le ton de l'humour»; mais, ce faisant, ses camarades lui signalent néanmoins clairement sa «dérive » vers la droite, son écart par rapport à « la ligne».

Moi-même j'ai été qualifié de droitier. Au début du NPA j'étais dans la majo, la plateforme A, Olivier Besancenot quoi... et au congrès de janvier 2011, je suis passé de la $P_{1}$ à la $P_{3}$, qui était la plateforme pour engager des discussions plus poussées avec le Front de gauche, pas encore pour y rentrer... et je suis rentré là et ça n'a pas traîné, dès les votes, localement et d'abord sur le ton de l'humour, sans méchanceté je leur en veux pas du tout... mais c'est une pratique militante aussi : «Ah, ça y est t'es un droitier maintenant!» Ceux-là et puis les copains dans d'autres villes, quand ils ont su que j'étais $P_{3}$, le petit texto de blague «Salut, t'es un droitier! »... Quand je suis monté au congrès, pareil "Salut, sale droitier!» Parce que la plupart de mes copains étaient restés en $P_{1}$. Bon, j'en avais quelques-uns en $P_{2}$ aussi, hein! (Rires) Mais bon, c'est vrai que surtout chez nous, «droite», ça a un petit côté vexant quand même... Et j'ai déjà vu des primo-militants, qui étaient pas au NPA au début, qui n'étaient pas à la LCR avant, être ultra-choqués par le courant CRI qui leur disait : "vos positions sont droitières», et eux ils pensaient qu'on les accusait d'être de droite! 24

Les deux animateurs du blog ont alors souhaité «s'emparer directement du qualificatif» droitier. Il s'agissait pour eux de le revendiquer en pratiquant "l'antiparastase», autrement dit en adoptant une stratégie de retournement du stigmate. La référence à l'anarchisme (" anarcho-droitier») vient renforcer, par le contraste de l'oxymore 25 , le caractère ironique de l'appellation 26 tout en manifestant leur attachement aux principes libertaires, partagés et revendiqués par une partie des militants et des dirigeants du NPA27.

Dans leur démarche, cette réappropriation de «l'insulte préférée de tout militant qui veut clore une question qu'il ne trouve pas suffisamment révolutionnaire à son goût „28 est une manière de chercher à dépasser des oppositions jugées stériles (anticapitalistes/antilibéraux, révolutionnaires/réformistes). Bien que critique et offensive, comme en témoigne le titre du blog, l'intention reste constructive et toujours motivée par le projet originel du NPA.

24. Guillaume, 35 ans, enseignant contractuel, entretien du 18 juillet 2013.

25. Qui n'en est pas complètement un, en réalité, puisqu'il existe des « anarchistes de droite » (qui défendent une conception libérale, au sens politique et économique, et aristocratique de l'anarchisme; Richard, 1997). Guillaume explique d'ailleurs que cela a pu parfois prêter à confusion, à l'intérieur comme à l'extérieur du NPA.

26. Si les droitiers sont considérés, par définition, comme plus à droite que les tenants de la ligne au sein d'une organisation d'extrême gauche, les «anarcho-communistes» (ou communistes libertaires) sont réputés être plus à gauche encore. En se nommant «anarcho-droitier», ils se situent donc symboliquement de part et d'autre de ceux qui les critiquent et qu'ils critiquent en retour.

27. Voir par exemple le texte d’Olivier Besancenot, «Pour un enrichissement libertaire du communisme», Contretemps, 2011, nº 4 .

28. "Charte du courant anarcho-droitier», billet du 31 juillet 2011. 
D'où l'idée (géniale) du Fight Club : nous sommes entre camarades et on va se taper dessus. Vous allez voir : ça fait un bien fou. Ainsi, il y aura des articles de réflexion, d'études [sic] et il y aura des billets gratuitement méchants. N'est-ce pas jouer contre son camp? Non. Notre démarche est celle d'un médecin qui décrit les symptômes d'une maladie. C'est dur et sans pitié, mais c'est nécessaire. Au malade, ensuite, d'adopter un comportement sain s'il veut survivre et avoir une descendance.

Si l'irrévérence et l'impertinence, qui seront la signature des anarcho-droitiers, en blessent certains, tant mieux. Qu'ils se disent que «tout ce qui ne nous tue pas nous rend plus fort » et qu'ils prennent du recul sur leur façon de militer. Si nos caricatures et nos vannes de potaches les énervent, qu'ils les réutilisent contre nos adversaires communs.

Enfin, nous avons l'intention de poursuivre avec tous ceux qui le souhaitent le projet initial du NPA, à savoir « rassembler le meilleur des différentes traditions du mouvement ouvrier».

Nous inviterons donc d'autres courants du mouvement ouvrier - révolutionnaires ou réformistes - à descendre dans notre cave pour voir ce qu'ils ont dans le ventre.

Nos adversaires seront ceux qui ne rigoleront pas à nos blagues.

Ça va chier! 29

C'est donc sur le ton de l'humour, un humour caustique inspiré de la presse satirique (Hara-Kiri et Charlie Hebdo), que ces deux « médecins » du NPA entreprennent de soigner leur parti «malade»30, en s'attaquant méthodiquement aux totems et emblèmes de l'extrême gauche.

\section{Contre les «curés rouges » et le «catéchisme révolutionnaire»}

Dans leurs billets, les animateurs du blog dénoncent régulièrement, avec une ironie parfois féroce, les travers des militants qu'ils qualifient de «curés rouges ", et s'en prennent à tous les symboles classiques de l'extrême gauche: les chants, les drapeaux, les slogans, etc.

Les «curés rouges» sont, au sein du NPA, les adversaires principaux des anarcho-droitiers. L'expression désigne selon les auteurs les gardiens du temple, défenseurs autoproclamés du marxisme, du léninisme et du trotskisme, autrement dit, les garants de la ligne révolutionnaire de l'anticapitalisme. À l'image des membres de la tendance CLAIRE, qui en forment en quelque sorte l'incarnation idéal-typique, les curés rouges sont les militants qui justifient toutes leurs prises de position en s’appuyant sur les «pères fondateurs»

29. «Mais c'est qui ces connards? », billet mis en ligne le 22 août 2011.

30. La métaphore médicale n'est pas sans rappeler le célèbre texte de Lénine sur le gauchisme comme «maladie infantile du communisme», dans l'ouvrage du même nom (voir note 1). 
du marxisme révolutionnaire, dont ils citent abondamment les œuvres. À l'inverse, toujours dans la provocation, les « anarcho-droitiers» revendiquent des références pour le moins iconoclastes : «plus Audiard que Trotsky, plus HaraKiri, Charlie Hebdo ou Le plan B que le programme de transition de 1938 «31.

Dans un billet intitulé «Ce qu'est un curé rouge », publié le 19 août 2011, les animateurs du blog en dressent un portrait précis, filant jusqu'au bout la métaphore du «catéchisme révolutionnaire» : le curé rouge se réfère aux «textes sacrés », « voue un culte aux saints et aux héros », " a parfois besoin d'un grigri qui le rassure » (un badge, une étoile rouge, un sigle antifasciste). Il «pratique l'incantation [...] rappelant fortement le credo catholique» et «a besoin d'un cérémonial». Plusieurs publications s'attaquent ainsi au «folklore révolutionnaire ». C'est le cas par exemple du billet «Changer de disque », qui propose la suppression du chant collectif L'Internationale, qualifiée de «chant religieux», à la fin des meetings, ou de celui intitulé «Changer de draps», qui propose d'ajouter un drapeau vert et un drapeau noir, couleurs de l'écologie et de l'anarchisme, au traditionnel drapeau rouge des révolutionnaires communistes 32 .

Les auteurs raillent également certaines habitudes vestimentaires (comme le port du keffieh) 33 et langagières (comme l'utilisation de sigles ou de jargon militant) 34 des militants " gauchistes ». Chaque billet est une manière de pousser plus loin, jusqu'à l'absurde, la revendication d'un positionnement droitier, en multipliant les blasphèmes afin de provoquer le débat sur le fond et sur la forme de l'anticapitalisme.

\section{La réception du blog: "ils nous prenaient carrément pour des ennemis»}

Après deux ans d'exercice, les deux militants dressent un bilan mitigé de l'expérience du blog. Ils ont bien été entendus, comme en témoigne la fréquentation du site et les réactions qu'il a suscitées, mais ils ne sont pas parvenus à redresser leur image et à faire reconnaître la légitimité de leur positionnement politique.

31. "Charte du courant anarcho-droitier» (voir supra note 22).

32. Sur les usages des couleurs en politique, voir notamment Pastoureau et Simonet (2005) et Bonnet, Constantin de Chanay et Desmarchelier (2014).

33. " [C] e colifichet nous vient tout droit du très saint royaume hachémite de Jordanie. À tout militant gauchiste ou propalestinien qui vous dira que "les seuls vrais keffiehs et ben y sont noir et blanc, ou alors si t'es un marxiste pro-FPLP tu peux à la rigueur en porter un rouge et blanc parce que j'te f'rai dire gnagnagna et gnagnagna..." hochez la tête, souriez mais ne dites rien, ça l'énervera ». L'auteur invite à porter un keffieh bleu, « des nuances de bleu UMP allié à un blanc immaculé (car régulièrement lavé à 40) ». «Anarcho-droitier fashion », billet du 19 juin 2013, 〈http:// fightclubnpa.blogspot.fr/2013/o6/anarcho-droitier-fashion.html〉.

34. Un billet « lexique » définit ainsi de manière humoristique une dizaine de termes du jargon militant, par exemple : «Soc-dem. : double troncature pour “social-démocrate”. Injure très puissante dans les milieux d'extrême gauche. Si vous êtes en face d'un gauchiste de $130 \mathrm{~kg}$, insultez plutôt la vertu de sa maman, ça passera mieux. syn. : traître, droitier, mou du genou ». "Lexique IV », billet du 18 octobre 2012, 〈http://fightclubnpa.blogspot.fr/2012/10/lexique-iv.html». 
URL d'origine des lecteurs du blog

Source : graphique tiré des statistiques du blog «Fight Club», juillet 2013

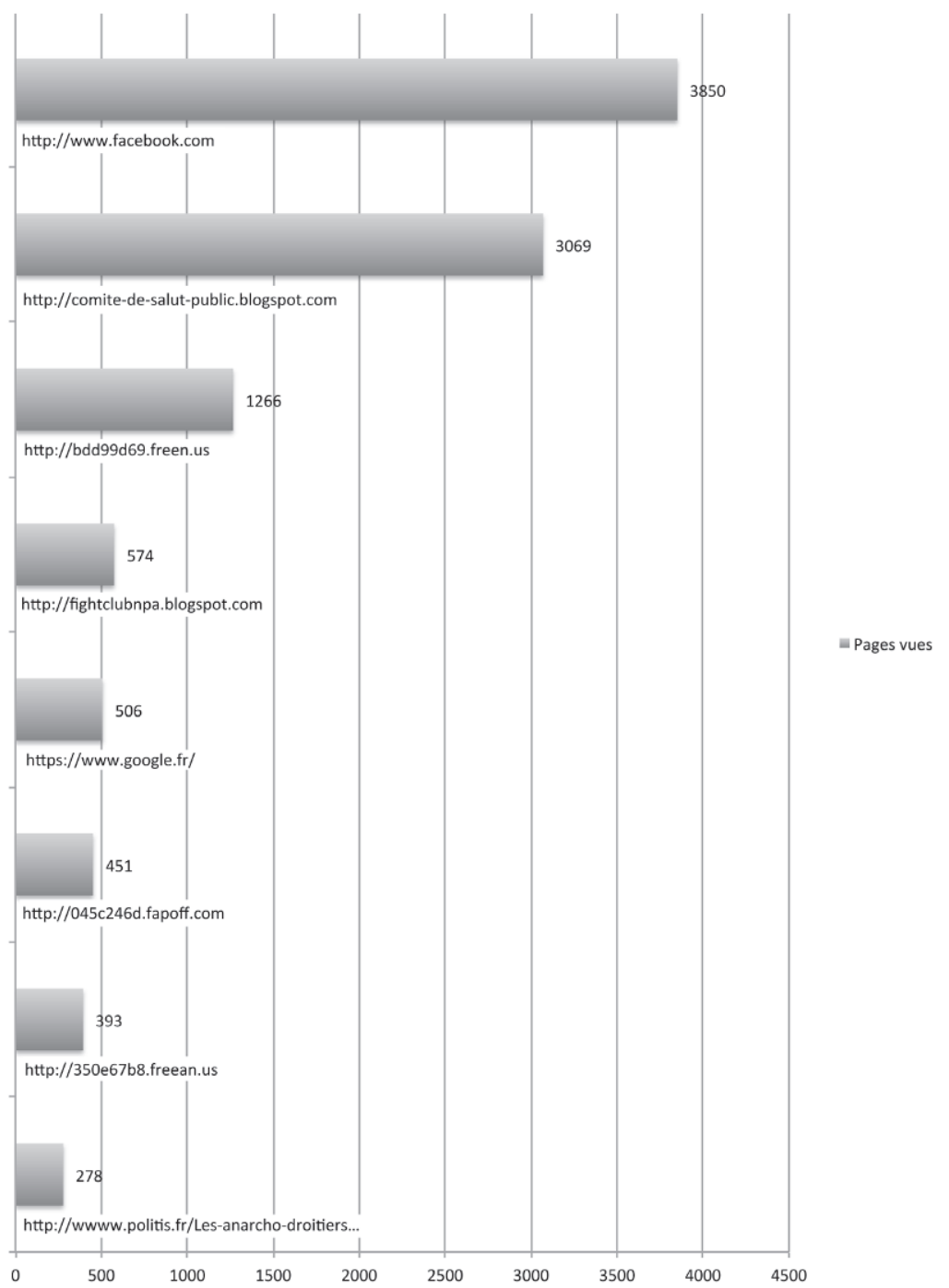

Le blog a connu, surtout dans sa première année, une très forte fréquentation (un peu plus de 160000 vues entre 2011 et 2013). Au maximum, au moment de son lancement, environ 12000 connexions par mois ont été enregistrées35; on en compte environ trois fois moins deux ans plus tard.

35. Les statistiques fournies par le site ne permettent pas de différencier le nombre de vues du nombre de visiteurs, donc de savoir combien de personnes ont généré ces 12000 connexions. 
La présence du blog sur les réseaux sociaux (notamment Facebook) et son signalement dans plusieurs journaux proches des milieux militants (comme Mediapart ou Politis) leur ont permis d'élargir un peu leur lectorat, comme le montre le relevé des URL d'origine des internautes.

Comme on le voit, la plupart des lecteurs ont accédé à un billet du blog via Facebook (3850 entrées), le "Comité de salut public », autre blog satirique animé lui aussi par un membre du NPA (3069 entrées), ou directement depuis une autre page du blog (574 entrées). Mais la publication de l'article consacré au «Fight Club » dans Politis a généré à elle seule 278 connexions directes (via un lien hypertexte) ${ }^{36}$.

Mais la lecture des commentaires des billets confirme que le blog est aussi fréquenté par des membres du NPA. Certains font part de leur amusement, acquiescent, parfois renchérissent dans la dénonciation ironique de tel ou tel travers militant et surtout saluent une «initiative salutaire». D'autres, à l'inverse, manifestent avec hostilité leur opposition à cette initiative, tant sur le fond que sur la forme. Les critiques parviennent aux auteurs via la fonction commentaire du blog et, plus rarement, directement par message électronique. Les animateurs constatent ainsi rapidement que le blog parle surtout aux convaincus et que cet usage de l'humour ne permet pas d'améliorer leurs relations avec les membres des courants « révolutionnaires», bien au contraire.

Les réactions, parfois hostiles, de la part des militants du parti révèlent en effet l'échec de la stratégie de retournement du stigmate. Leur effort critique est perçu comme un «travail de sape » et la revendication du qualificatif droitier n’a fait que renforcer le caractère politiquement déviant des auteurs du blog.

Mais on a vu ils en avaient rien à foutre puis qu'ils nous prenaient carrément pour des ennemis, pour des gens qui voulaient détruire le NPA, alors que c'était pas ça du tout, vraiment... C'était surtout des gens qui tournaient autour de la plateforme, qui ne comprenaient pas notre démarche, qui nous accusaient d'être des sousmarins du Front de gauche, alors que pas du tout, on était très réticents à l'époque, on était encore très critiques là-dessus...37

Le fait d'être considérés non pas « comme des camarades mais comme des ennemis » achève de les éloigner du parti. Le coauteur du blog quitte le NPA après le congrès de 2011 pour rejoindre le Front de gauche en adhérant au Parti de gauche. Guillaume quitte lui aussi le NPA peu de temps après, mais milite au

36. On peut faire néanmoins l'hypothèse que les lecteurs de l'article de Politis qui ont ensuite cliqué sur le lien conduisant au «Fight Club » sont en partie des membres ou des proches du NPA, déjà informés de l'existence du blog ou prédisposés à s'y intéresser. On notera également que certaines URL (freean.us, frapp.off) ne correspondent pas à des lecteurs mais renvoient à de fausses références générées par des robots et créées pour amener les visiteurs vers des sites web indésirables (spamming).

37. Guillaume, 35 ans, enseignant contractuel, entretien du 18 juillet 2013. 
sein d'un petit comité local de la Gauche anticapitaliste38. Il maintient pendant quelques mois l'activité du blog (parce qu'il estime avoir encore «quelques comptes à régler») puis y met un terme, provisoire, le 11 septembre 2013 («une date facile à retenir »). Il publie encore, de manière plus sporadique, quelques billets relayés sur une page Facebook, dans lesquels il s'attaque également, désormais, aux travers du Front de gauche.

\section{Recompositions de l'extrême gauche et pratiques du pluralisme intrapartisan}

Pour conclure, l'analyse montre que l'usage du terme droitier au sein de l'extrême gauche vise à produire une sorte de topographie politique des positions tenues par les militants et surtout à alimenter des luttes de position, et donc des luttes de pouvoir, à l'intérieur du parti. Le terme droitier constitue, comme on l'a montré, une incitation négative aux tentations «réformistes » et aux écarts supposés avec une stratégie pensée comme «révolutionnaire». Le risque de la stigmatisation favorise une forme d'orthodoxie militante dans la mesure où les tentatives de "prise de parole» (Hirschman, 1995) discordantes donnent lieu à un rappel de la doxa («la ligne») visant d'abord la conversion et conduisant, en cas d'échec, à une mise à l'écart (du côté des «ennemis»). Cette difficulté à faire entendre des analyses de la situation politique et des stratégies politiques alternatives à une certaine lecture du modèle trotskiste a conduit de nombreux militants à préférer la défection, alors même que les positions qu'ils défendaient n'étaient pas nécessairement plus minoritaires (si l'on en croit le nombre de départs des militants dits " unitaires ») que celles des courants qui se présentent comme révolutionnaires.

Au-delà de la bataille sémantique, l'analyse des relations entre les courants qui composent le NPA, par le prisme des usages du qualificatif droitier, met donc au jour les difficultés rencontrées lors de cet «aggiornamento manqué» (Matonti, 1999). La volonté initiale d'ouverture à l'ensemble des traditions contestataires a en effet rapidement cédé la place à une concurrence interne dont l'objectif n'était pas la production d'une «nouvelle idéologie», qui ferait la somme, sinon la synthèse, des expériences passées et des courants existants au sein du mouvement ouvrier, mais bien l'imposition d'une certaine conception de la pratique politique. L'importance de la «culture politique » à l'extrême gauche, la forte socialisation politique de ses membres (au

38. La «gauche anticapitaliste - courant unitaire pour l'écosocialisme » est un courant né au sein du NPA en novembre 2011 dans le prolongement de la « $P_{3}$ » du congrès de 2011. Ce courant du NPA a rejoint le Front de gauche en juillet 2012 avant de fusionner, en novembre 2013, avec d'autres composantes (Convergences et Alternative et la «Gauche unitaire », également issues du NPA, et la Fédération pour une alternative sociale et écologique) pour former Ensemble! - Mouvement pour une alternative de gauche, écologiste et solidaire. 
moins de ses «cadres» au niveau national comme local), formant un habitus politique collectif relativement dense, contribuent peut-être à expliquer cette inertie des cadres de l'action collective partisane.

D’un point de vue plus général, ce cas permet d'interroger la gestion du pluralisme intrapartisan, selon les termes de Giovanni Sartori (1976). Le NPA permet certes l'institutionnalisation des tendances, leur libre formalisation idéologique et leur donne des moyens d'expression (tribunes dans le journal du parti, contribution au financement d'une publication propre, participation aux frais liés aux réunions et aux activités de la tendance, etc.). Mais cette assurance statutaire du droit aux divergences doit être mise en relation avec les conditions pratiques du maintien de l'hétérogénéité au sein du groupe. En l'occurrence, on observe que le jeu des tendances, valorisé comme un impératif de démocratie interne, conduit à l'élimination progressive des courants jugés déviants par rapport à la conception dominante de l'action politique. Formellement, aucun courant n'a été exclu du parti et les sortants ont toujours fait de leur décision un choix positif. Pour autant, l'analyse ne doit pas manquer l'effet des interactions intrapartisanes sur les choix des militants : la désignation de droitier, assimilée à une insulte et fonctionnant comme l'affirmation publique d'une déviance, rend, en pratique, quasi impossible le maintien des engagements. Cette insulte, qui fonctionne comme acte de destitution dans la mesure où elle renvoie à la fois à l'histoire sédimentée de cette organisation partisane et plus largement de l'extrême gauche et aux interactions militantes qui l'agitent, revêt alors un caractère performatif, « qui réalise dans le présent des mots un effet futur » (Bourdieu, 2001, p. 111) : elle conduit à la défection les militants dont le positionnement est assimilé à une « dérive».

\section{Références}

AebISCHEr Sylvie, 2003, Gauche-Droite. Au-delà de cette limite la politique n'est plus pensable, Paris, Nicolas Philippe.

BARBET Denis, 2002, "Parti et syndicat dans l'espace léninien. Les mots de Que faire?», Mots. Les langages du politique, n68, p. 9-26.

Bensaï̀ Daniel, 2002, Les trotskismes, Paris, PUF. BouRdieu Pierre, 1984, «La grève et l'action politique », Questions de sociologie, Paris, Minuit.

- 2001, Langage et pouvoir symbolique, Paris, Le Seuil.

- 2003 [1997], Méditations pascaliennes, Paris, Le Seuil.

Bonnet Valérie, Constantin de Chanay Hugues, Desmarchelier Dominique, 2014, «Des couleurs et de la politique», Mots. Les langages du politique, nº 105, p. 5-11.

CRAPEZ Marc, 1998, «De quand date le clivage gauche/droite en France? », Revue française de science politique, vol. 48, $\mathrm{n}^{\circ} 1, \mathrm{p} .42-75$.

DE WAELE Jean-Michel, SEILER Daniel-Louis, 2011, Les partis de la gauche anticapitaliste en Europe, Paris, Economica. 
Dubar Claude, 2000, La socialisation. Construction des identités sociales et professionnelles, Paris, Armand Colin.

Dunezat Xavier, 2008, "La division sexuelle du travail militant dans les assemblées générales. Le cas des mouvements de "sans" », Amnis. Revue de Civilisation Contemporaine Europes/Amériques, n 8, 〈http://amnis.revues.org/524> (consulté le 6 juin 2016)

Fillieule Olivier, Mathieu Lilian, Roux Patricia éd., 2007, « Militantisme et hiérarchies de genre », Politix, nº 78 .

GAïTı Brigitte, 2001, «Les inconstances politiques », Politix, nº 14, p.17-42.

GAUCHET Marcel, 1997 [1992], «La droite et la gauche», Les Lieux de mémoire, t. III, Les France, 1. Conflits et partages, P. Nora éd., Paris, Gallimard, p. 2533-2600.

GAXIE Daniel, 1977, «Économie des partis et rétributions du militantisme», Revue française de science politique, vol.27, $\mathrm{n}^{\circ}{ }^{1}$, p. 123-154.

- 2012, "Droite ou gauche? Usages et non-usages d'instruments courants d'orientation politique», Gauche/droite. Genèse d'un clivage politique, J.Le Bohec, C. Le Digol éd., Paris, PUF, p. 449-475.

Goffman Erving, 1974, Les rites d'interaction, Paris, Minuit.

- 1977, Stigmate. Les usages sociaux des handicaps, Paris, Minuit.

HIRSCHMAN Albert O., 1995, Défection et prise de parole, Paris, Fayard.

JohsuA Florence, 2015, Anticapitalistes. Une sociologie historique de l'engagement, Paris, La Découverte.

LANUQUE Jean-Guillaume, 2005, "Les trotskystes et les insultes. Esquisse de typologie et d'interprétation », L'Insulte (en) politique. Europe et Amérique latine du XIXe siècle à nos jours, T. Bouchet, M. Legget, J. Vigreux, G. Verdo éd., Dijon, Éditions universitaires de Dijon, p.169-176.

LE BoHeC Jacques, LE DIGOL Christophe, 2012, Gauche/droite. Genèse d'un clivage politique, Paris, PUF. Lindenberg Daniel, 2004, "À gauche de la gauche», Histoire des gauches en France, t. II : xxe siècle. À l'épreuve de l'histoire, J.-J. Becker, G. Candar éd., Paris, La Découverte, p.119-134.

MAtontı Frédérique, 1999, Les intellectuels communistes. Essai sur l'obéissance politique. «La Nouvelle Critique » (1967-1980), Paris, La Découverte.

OLSON Mancur, 1978, Logique de l'action collective, Paris, Fayard.

Pastoureau Michel, Simonnet Dominique, 2005, Le petit livre des couleurs, Paris, Le Seuil.

RICHARD François, 1997, Les anarchistes de droite, $2^{\mathrm{e}}$ édition, Paris, PUF, coll. Que saisje?

RICHARD Gilles, 2006, "Droites et gauches dans la vie politique française. Réflexions sur un affrontement pluriel », Vingtième siècle. Revue d'histoire, nº 9o, p.155-167.

SARtori Giovanni, 1976, Parties and Party System, Cambridge, Cambridge University Press.

Werth Nicolas, 2006, Les Procès de Moscou : 1936-1938, Bruxelles, Complexe. 
